\title{
Cisto neuroentérico espinhal: relato de um caso e revisão da literatura
}

\section{Spinal Neuroenteric Cyst: Report of a Case and Review of the Literature}

\author{
Cléciton Braga Tavares ${ }^{1}$ Emerson Brandão Sousa ${ }^{2}$ Igor Brenno Campbell Borges ${ }^{3}$ \\ Amauri Araújo Godinho Júnior ${ }^{4}$ Benício Oton de Lima ${ }^{4}$ \\ Francisca das Chagas Sheyla Almeida Gomes Braga ${ }^{5}$ \\ ${ }^{1}$ Neurocirurgião, Professor de Neurologia/Neurocirurgia da \\ Faculdade de Ciências Médicas, Universidade Estadual do Piauí \\ (FACIME/UESPI), Teresina, Piauí, Brasil \\ 2 Mestrado, Neurocirurgião; Professor de Neurologia/Neurocirurgia \\ da FACIME/UESPI, Teresina, Piauí, Brasil \\ ${ }^{3}$ Neurocirurgião do Hospital de Base do Distrito Federal (HBDF), Brasília, Brasil \\ ${ }^{4}$ Neurocirurgião e Preceptor do Programa de Residência Médica em \\ Neurocirurgia do HBDF, Brasília, Brasil \\ ${ }^{5}$ Mestrado, Enfermeira do Hospital de Urgências de Teresina, \\ Teresina, Piauí, Brasil \\ Arq Bras Neurocir 2015;34:250-257.

\begin{abstract}
Address for correspondence Cléciton Braga Tavares, MD, Conjunto IAPEP, bloco C, apto 32, Ilhotas, Teresina, PI, Brazil, CEP 64015-040 (e-mail: bragatavares@yahoo.com.br).
\end{abstract}

\section{Resumo \\ Palavras-Chaves \\ - cisto enterogenous \\ - cisto neuroentérico \\ - tumor da medula espinhal \\ - disrafismo espinhal \\ - laminectomia}

Cistos neuroentéricos espinhais são anomalias do desenvolvimento, mais comumente encontrados no mediastino posterior e raramente presentes no sistema nervoso central. Geralmente localizados na coluna cervicotorácica. Principalmente encontrados em pacientes jovens e com predomínio no sexo masculino (2:1). Várias hipóteses têm sido sugeridas para explicar a embriogênese dos cistos neuroentéricos, entre elas a mais aceita seria uma incompleta separação entre o ectoderma e o endoderma. Manifesta-se principalmente como dorsalgia e déficit neurológico progressivo. Alguns pacientes apresentam surtos de dor e déficits transitórios. A ressecção completa da lesão, com esvaziamento do cisto e remoção do envoltório, é o tratamento de eleição. As principais complicações pósoperatórias são a recidiva da lesão, aracnoidite e piora do déficit neurológico.

Developmental abnormalities are more commonly found in the posterior mediastinum and rarely present in the central nervous system. Usually located in the cervical and thoracic spine. Mainly found in young patients with predominance in males (2:1). Several hypotheses have been suggested to explain the embryogenesis of neuroentéricos cysts, including the most accepted would be an incomplete separation between the ectoderm and endoderm. It manifests primarily as back pain and progressive neurological deficits. Some patients experience bouts of pain and transient deficits. Complete resection of the lesion with dissection of the cyst and removing the wrap, is the treatment of choice. The main postoperative complications are recurrence of injury, arachnoiditis and worsening of neurological deficit.
Recebido

July 7, 2014

Aprovado

March 31, 2015
DOI http://dx.doi.org/

$10.1055 / \mathrm{s}-0035-1554050$. ISSN $0103-5355$.
Copyright $(2015$ by Thieme Publicações License terms Ltda, Rio de Janeiro, Brazil $\circledast(1) \Theta$ 


\section{Introdução}

Cistos neuroentéricos espinhais são anomalias do desenvolvimento, inicialmente descritas por Puusepp em 1934. Mais comumente são encontrados no mediastino posterior e raramente estão presentes no sistema nervoso central. ${ }^{1,2}$

Várias hipóteses têm sido sugeridas para explicar a embriogênese dos cistos neuroentéricos, entre elas a mais aceita seria uma incompleta separação entre o ectoderma e o endoderma, ocorrendo à persistência de resíduos do endoderma no interior do neuroectoderma, durante a terceira semana do desenvolvimento embrionário. ${ }^{1-5}$ Há raríssimos relatos de cistos neuroentéricos intracranianos com disseminação liquórica para a coluna vertebral. ${ }^{3}$

Geralmente localizados na coluna cervicotorácica, podem ser justamedulares (95\% dos casos) ou intramedulares (5\% dos casos). Predominam na região anterior da medula espinhal e representam $0,7-1,3 \%$ de todos os tumores espinhais. Principalmente encontrados em pacientes jovens, apresentam média de idade de 25,8 anos e predomínio no sexo masculino (2:1). . $2,6,7^{-}$

\section{Relato do Caso}

Paciente RCS, sexo feminino, 30 anos de idade. Admitida no serviço de emergência do Hospital de Base do Distrito Federal, Brasília, com o relato de paraparesia progressiva há cerca de 20 dias e incontinência urinária.

Ao exame estava consciente e orientada, paraparética (força grau III), hiperreflexia de patelar e aquileu bilateralmente e clônus esgotável em membros inferiores. Hipoes- tesia tátil e dolorosa, com nível sensitivo em T2. Ataxia sensitiva, com sinal de Roomberg presente. Incontinência urinária.

A tomografia de coluna evidenciou deformidade em padrão de asa de borboleta e fusão dos corpos vertebrais de T1-T2 com aumento da cifose torácica neste nível. A ressonância mostrou a presença de uma lesão cística intrarraquidiana e extramedular na topografia de T1-T2, isointensa na ponderação T1 e hiperintensa na ponderação T2.

Foi submetida a laminectomia e exérese da lesão cística. Optou-se por artrodese torácica via posterior no mesmo tempo cirúrgico com o intuito de evitar uma possível instabilidade e aumento da cifose torácica no pós-operatório. $\mathrm{O}$ exame histopatológico confirmou tratar-se de um cisto neuroentérico. Após a cirurgia a paciente manteve o mesmo déficit neurológico da admissão, sendo acompanhada ambulatorialmente.

Após 11 meses da cirurgia apresentou quadro doloroso e piora do déficit neurológico, passando de paraparesia com força grau III para grau II. A ressonância de controle mostrou recidiva da lesão. Foi submetido a nova exérese da lesão. No pós-operatório apresentou paraplegia. Durante internação hospitalar evoluiu com fístula liquórica pela ferida operatória e meningite bacteriana, sendo tratada com acetazolamida, cefepime e vancomicina.

Atualmente, quatro anos depois, segue em acompanhamento ambulatorial, sem recidiva da lesão. Mantém-se paraplégica, com anestesia tátil e dolorosa (nível sensitivo em T2) e realizando cateterismo vesical intermitente para esvaziamento da bexiga.

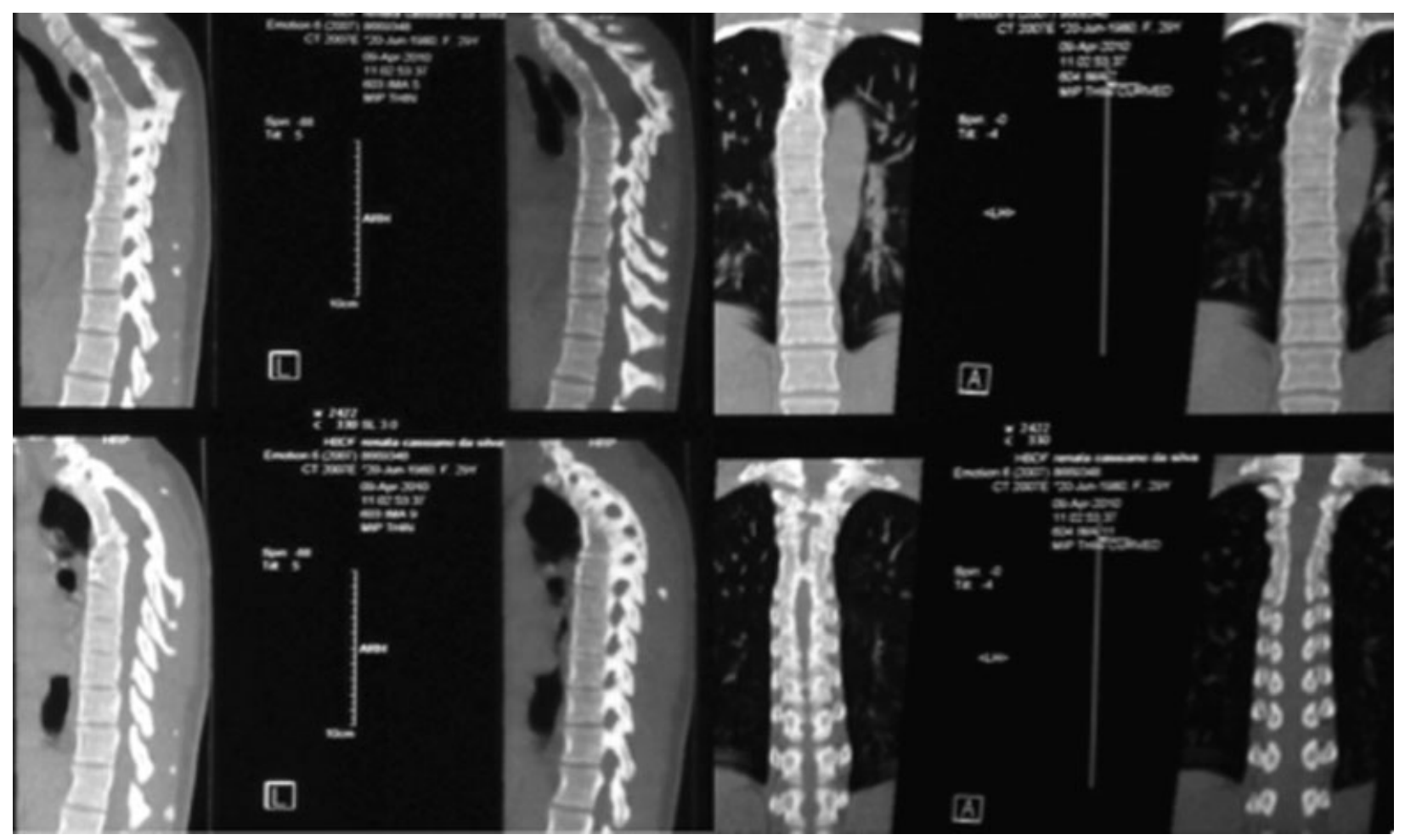

Fig. 1 Tomografia de coluna torácica mostrando malformação dos corpos vertebrais de T1e T2. 


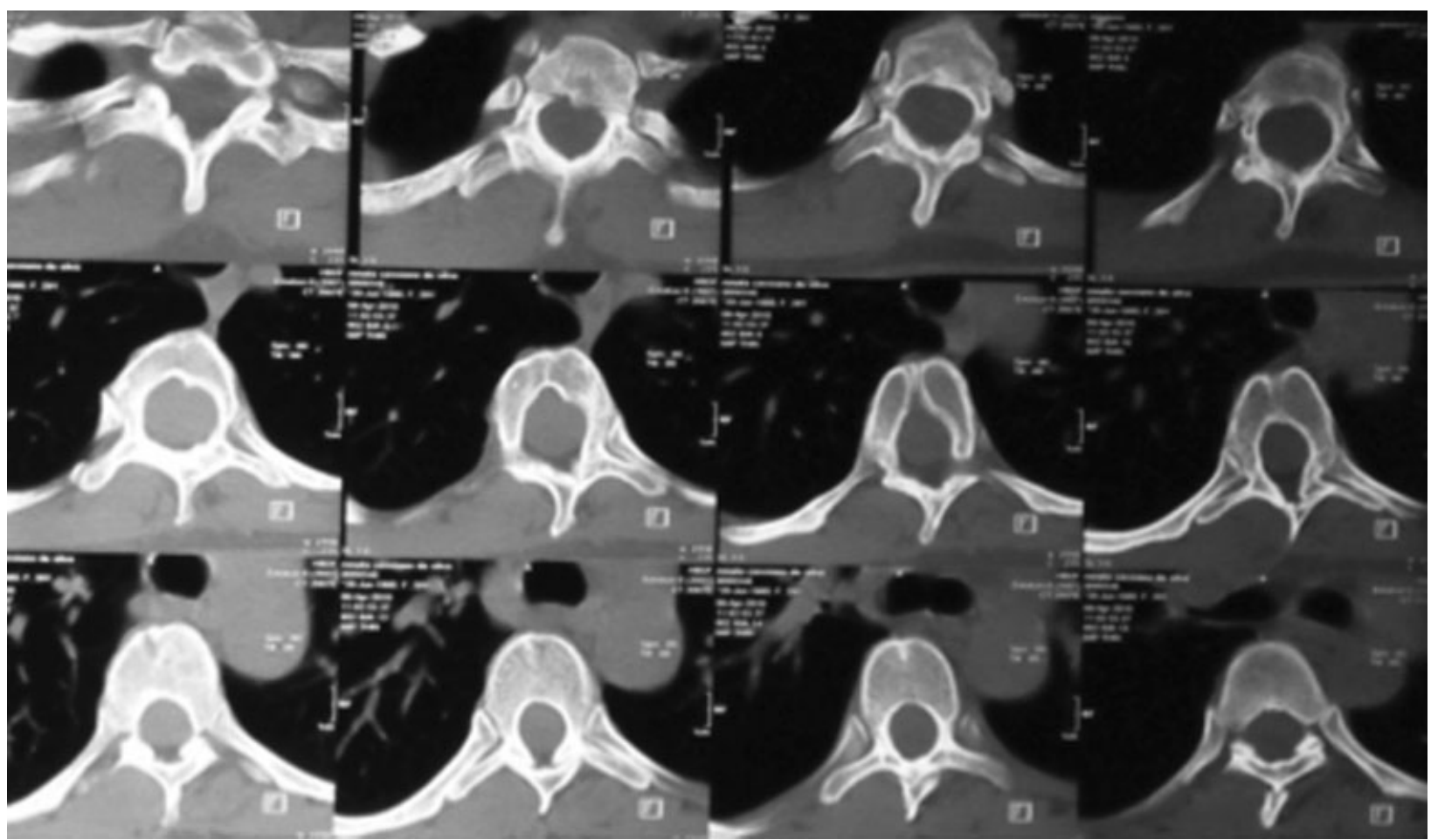

Fig. 2 Tomografia de coluna torácica em cortes axiais mostrando malformação dos corpos vertebrais de T1 e T2, tipo "asa de borboleta".

\section{Discussão}

A paciente deste relato iniciou o quadro com fraqueza progressiva nos membros inferiores e evolução de 20 dias. Manifestação semelhante à descrita pela literatura, que aponta a dorsalgia e o déficit neurológico progressivo como as formas iniciais de apresentação. ${ }^{1}$

Alguns pacientes apresentam surtos de dor e déficits transitórios. ${ }^{1}$ A queixa de dor ocorreu apenas durante a recidiva da lesão, cerca de 11 meses após a primeira cirurgia. Aracnoidite química, meningismo devido a infecção e ruptura cística já foram descritos como sendo responsáveis pelo quadro doloroso. ${ }^{2}$

A tomografia mostrou alterações e fusões dos corpos vertebrais de T1 e T2 (-Figs. 1 e 2). A frequente associação de defeitos vertebrais anteriores sugere fortemente que a origem dos cistos neuroentéricos está relacionada a defeitos de separação da notocorda durante a formação do canal alimentar. ${ }^{8}$ Entre os defeitos podemos destacar a espinha bífida anterior ou posterior, defeitos em asa de borboleta, diastematomielias, fusões de corpos vertebrais, hemivértebras e Klippel-Feil. ${ }^{7}$

Radiologicamente, são lesões não homogêneas, isointensas a levemente hiperintensas em $\mathrm{T} 1$, hiperintensas em $\mathrm{T} 2$ e sem realce ao meio de contraste. $\mathrm{O}$ sinal em T1 pode variar com a concentração de proteínas ou presença de hemorragias dentro do cisto. ${ }^{6,9}$ Nas imagens deste caso evidenciamos um isosinal em T1 e hipersinal em T2, tanto no início do quadro (-Figs. 3, 4 e 5) como na recidiva da lesão (-Fig. 6).

A paciente foi submetida a laminectomia e exérese do cisto (-Figs. 7 e 8). A ressecção completa da lesão, com

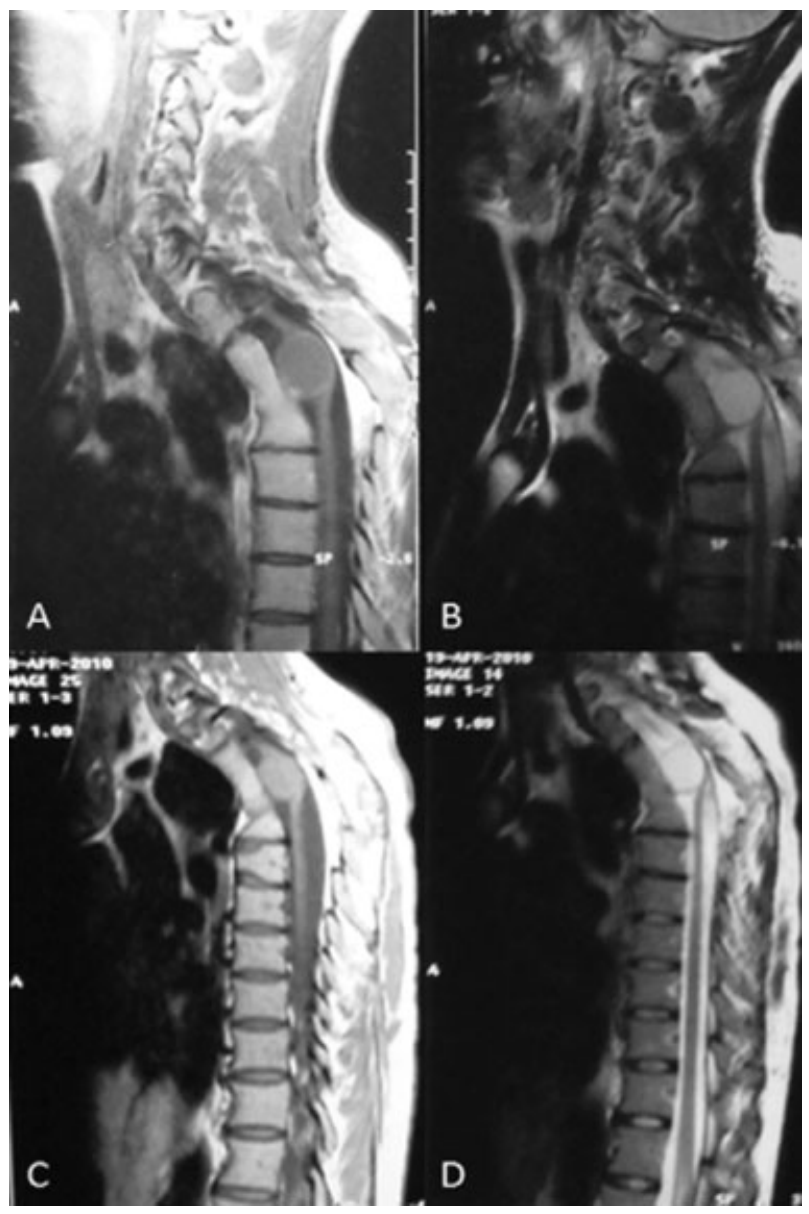

Fig. 3 Ressonância de coluna cervicotorácica evidenciando lesão intrarraquidiana e extramedular. (A) cervical T1, (B) cervical T2, (C) torácica T1, (D) torácica T2. 

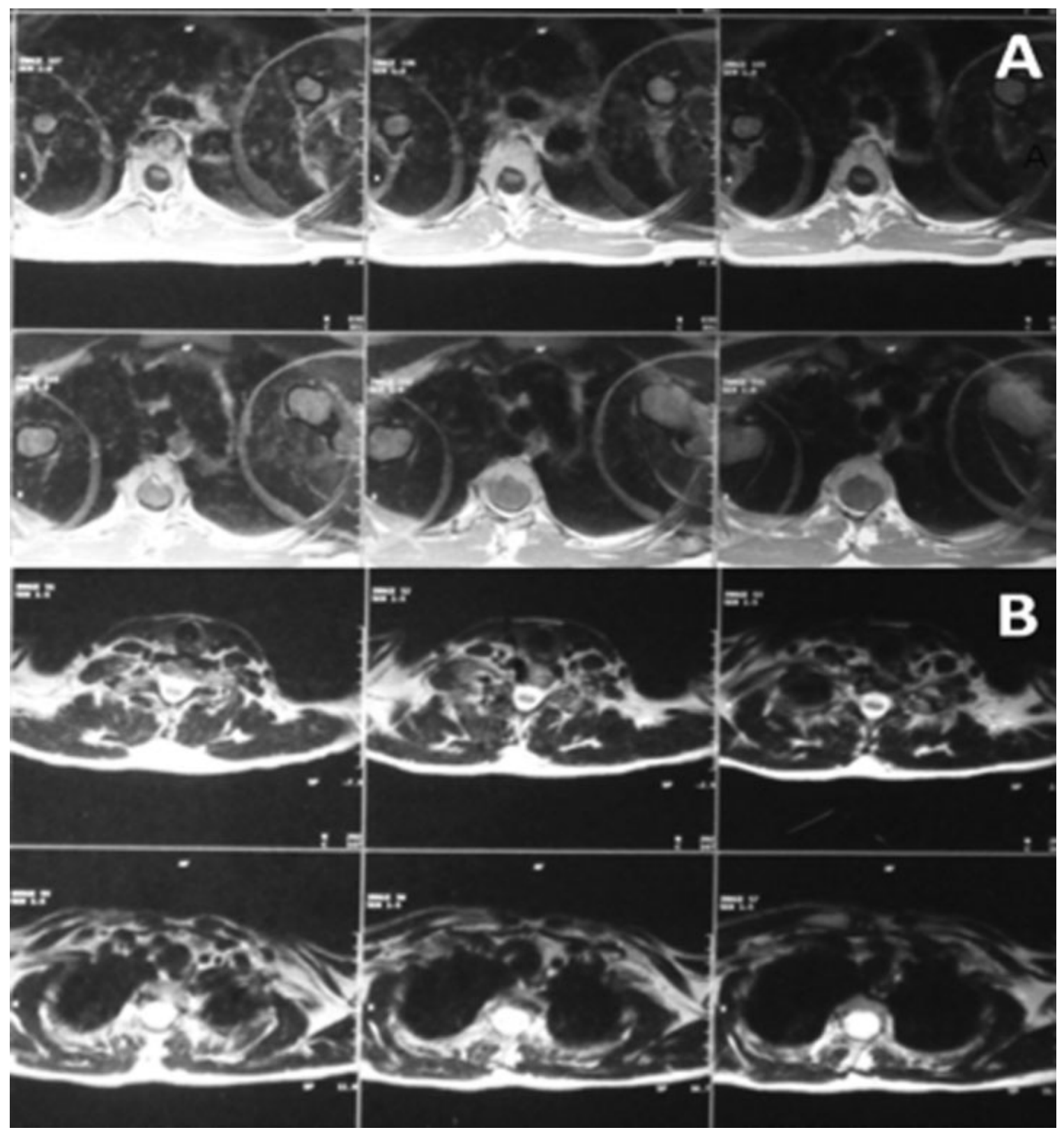

Fig. 4 Ressonância magnética de coluna torácica, em cortes axiais: (A) T1 sem contraste, (B) T2.

esvaziamento do cisto e remoção do envoltório, é o tratamento de eleição. $O$ acesso por via posterior é a opção de escolha na maioria dos casos, sendo possível a realização da laminectomia ou lamintomia. ${ }^{1,4,6}$ Optamos por realizar artrodese via posterior com receio de ocorrer uma instabilidade ou progressão da cifose no pós-operatório, uma vez que a coluna já apresentava deformidades e cifose acentuada ao nível do cisto.

Alguns autores realizam uma comunicação entre o leito da lesão e o espaço subaracnóideo após a completa remoção do cisto. ${ }^{2}$ Entretanto, a ressecção parcial é uma alternativa possível em lesões intramedulares, devido à ausência de um plano de clivagem adequado, ou quando há grande aderência do cisto extramedular com a medula espinhal. ${ }^{1,2,6}$

Após esta primeira cirurgia não houve melhora do déficit motor, a paciente manteve-se paraparética com força grau III.
A melhora neurológica é dependente da idade do paciente, duração dos sintomas e do grau de descompressão medular. ${ }^{2}$

$\mathrm{O}$ estudo patológico evidenciou tratar-se de um cisto neuroentérico. Histologicamente, são cistos envoltos por epitélio colunar, ciliado ou não, pseudoestratificado e com grânulos de mucina em seu interior. ${ }^{5,6,10} \mathrm{~A}$ parede do cisto é positiva para citoqueratina, EMA e CEA e negativa para $\$ 100 \mathrm{e}$ GFAP. ${ }^{7,11}$

Apesar de consideramos ressecção completa na primeira cirurgia, 11 meses após houve recidiva da lesão, com dorsalgia e piora da fraqueza nos membros inferiores. As principais complicações pós-operatórias são a recidiva, aracnoidite e piora do déficit neurológico. ${ }^{4}$ A recorrência do cisto pode acontecer devido ao acúmulo de líquor cefalorraquidiano por osmose ou à produção de um exsudato pela parede cística residual. ${ }^{9}$ 
254 Cisto neuroentérico espinhal Tavares et al.

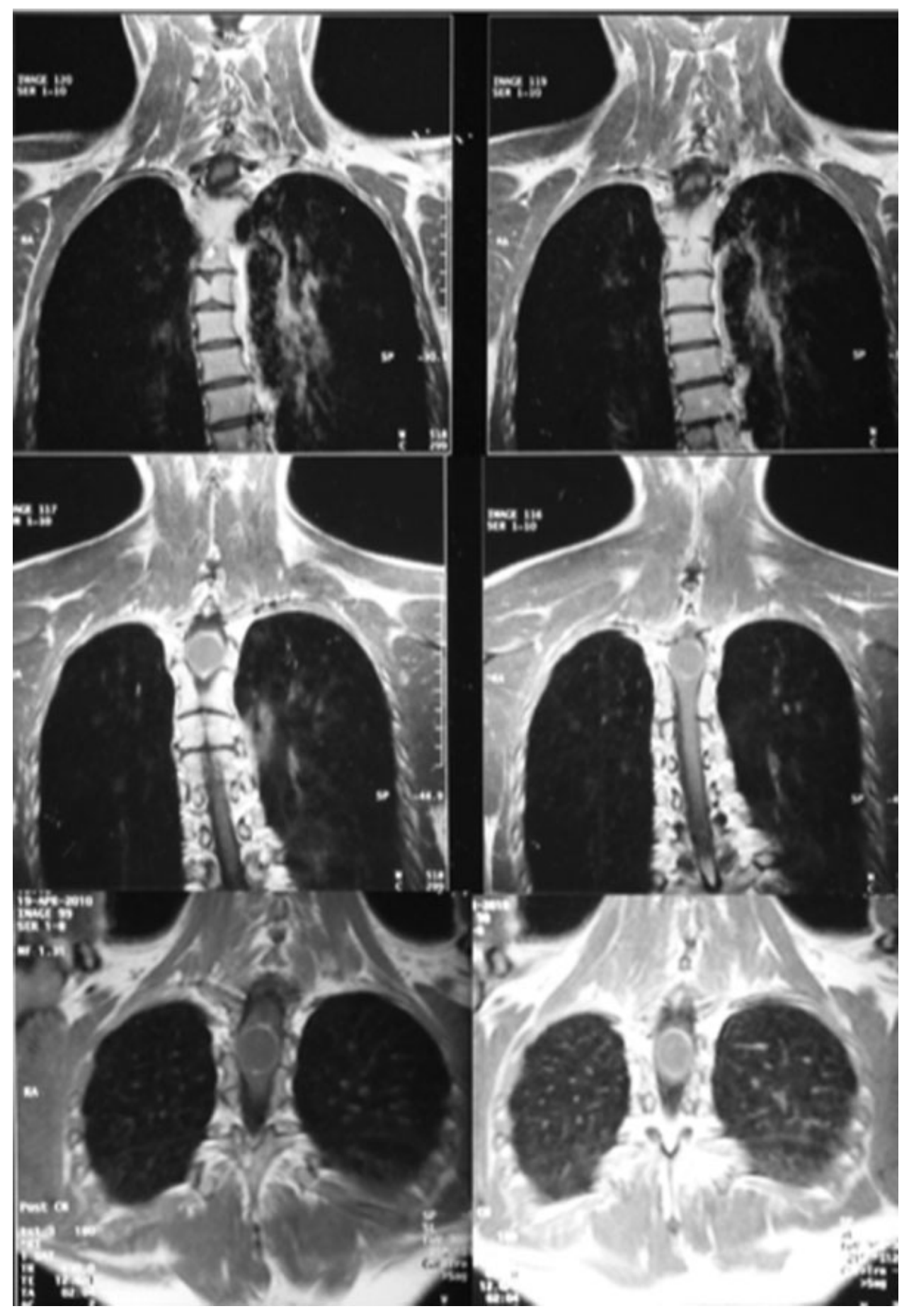

Fig. 5 Ressonância magnética de coluna torácica em cortes coronais. 


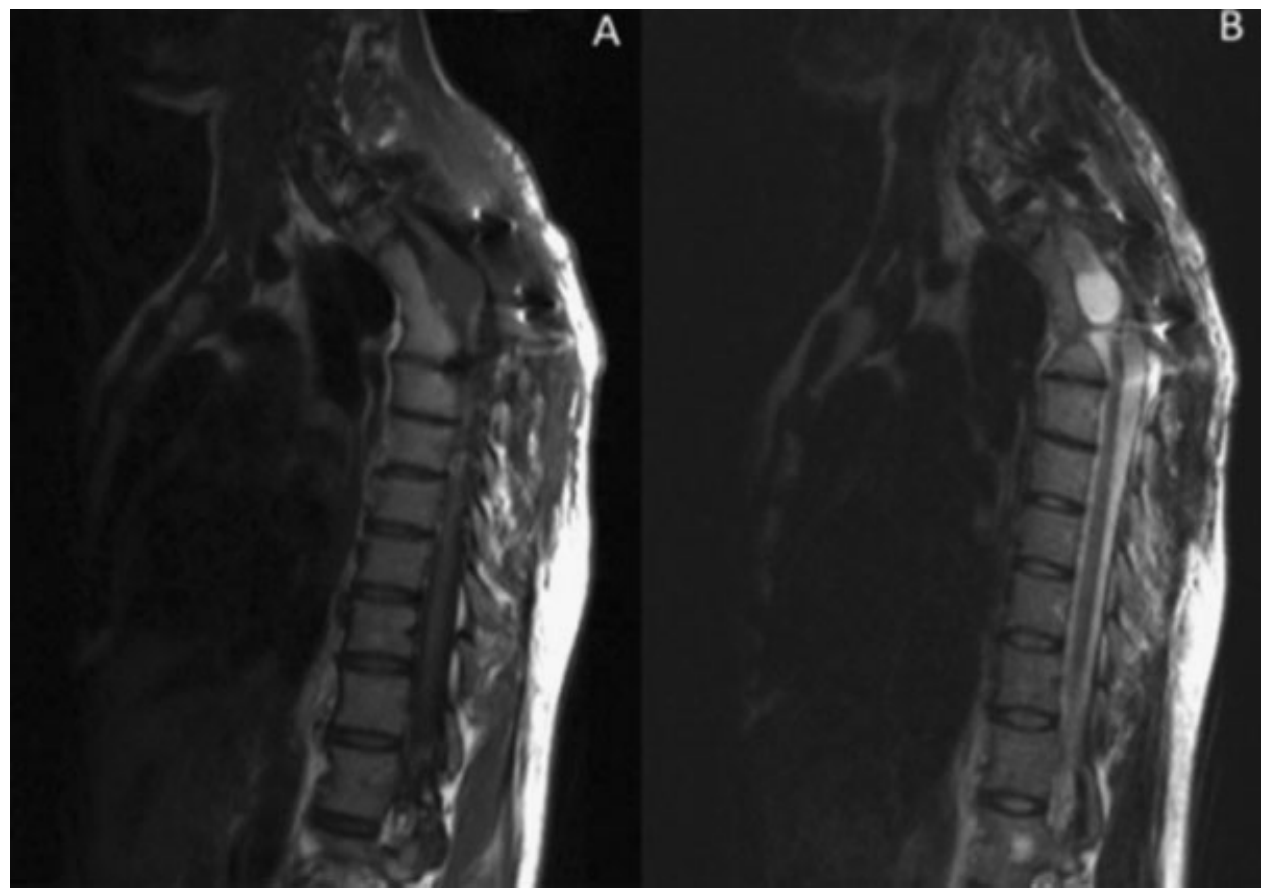

Fig. 6 Ressonância magnética sagital da coluna torácica. Recidiva da lesão. (A) Ponderação T1, (B) ponderação T2.

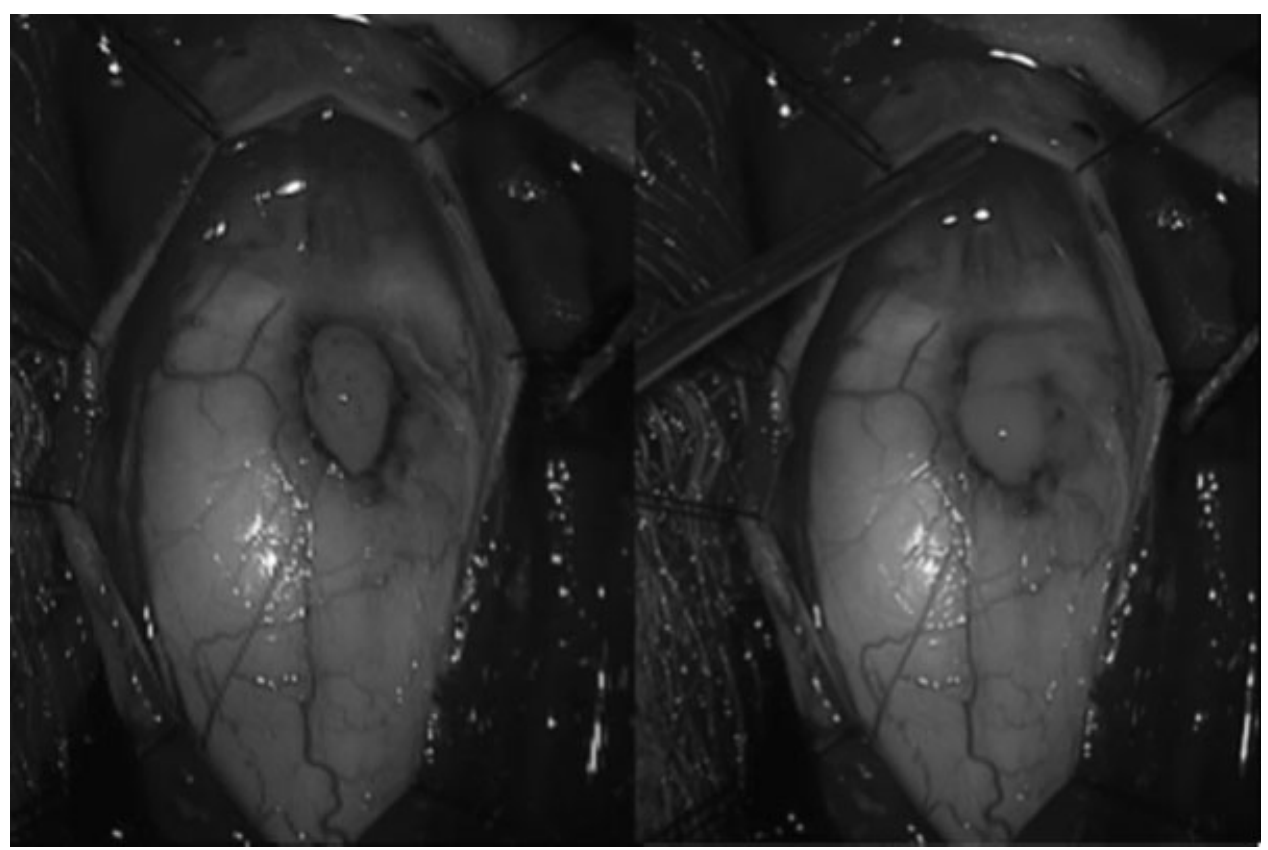

Fig. 7 Laminectomia + durotomia e exposição da lesão cística. Primeira abordagem cirúrgica. 


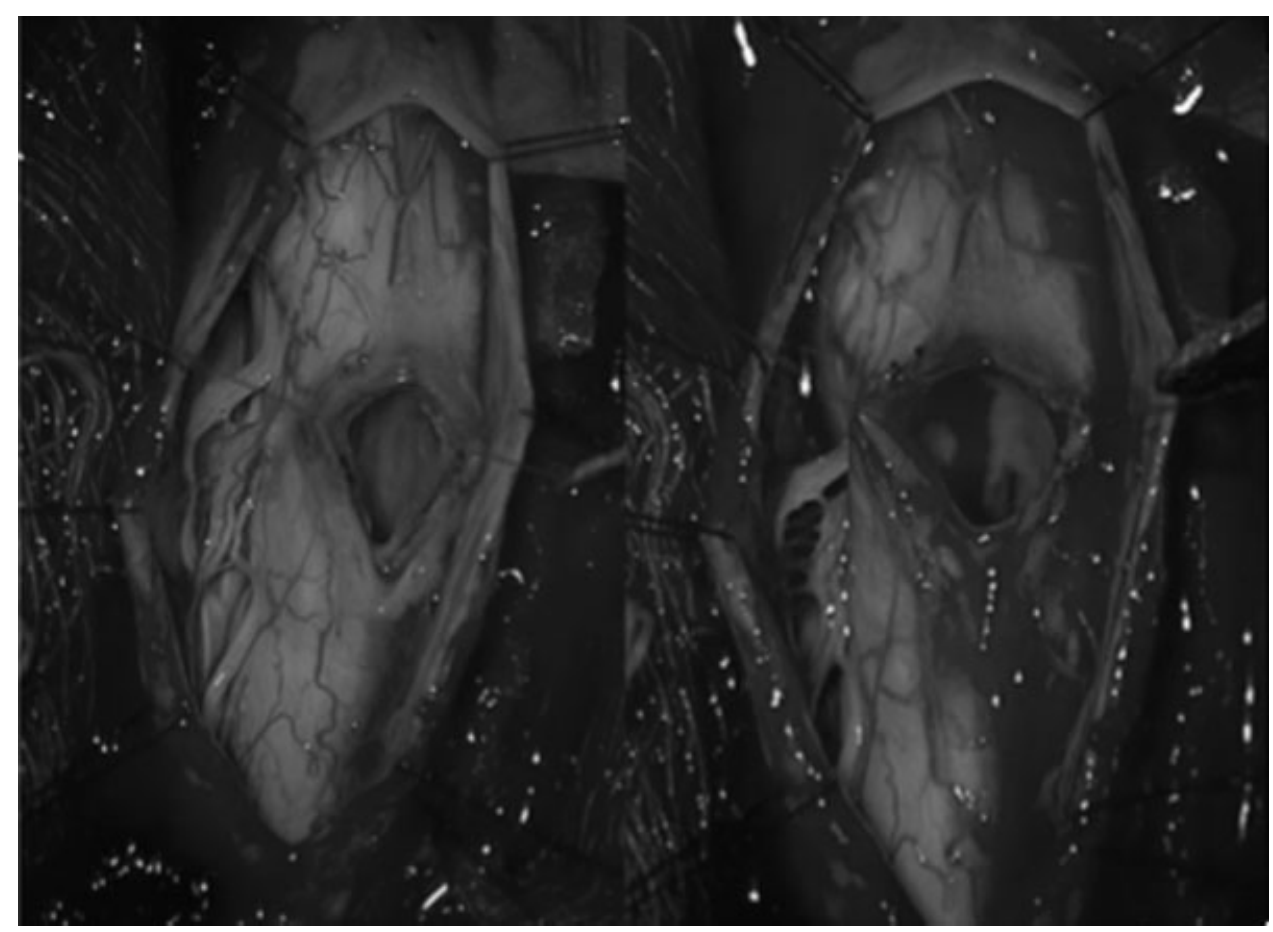

Fig. 8 Aspecto medular após a exérese da lesão cística. Primeira abordagem cirúrgica.

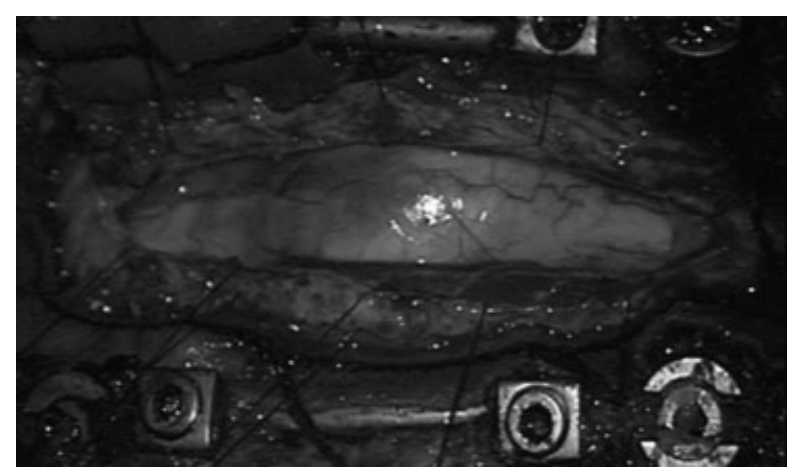

Fig. 9 Durotomia e exposição da lesão cística. Segunda abordagem cirúrgica.

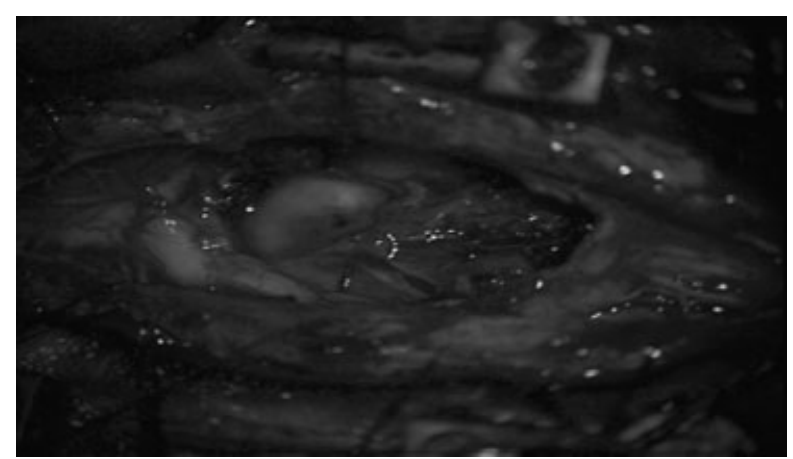

Fig. 10 Aspecto medular após a exérese da lesão cística. Segunda abordagem cirúrgica.
A paciente realizou nova exérese da lesão ( - Figs. 9 e 10). A ressecção parcial está associada a uma maior taxa de recidiva, mas os trabalhos evidenciam que não há um pior prognóstico, nem mesmo após uma segunda intervenção. ${ }^{1}$ No entanto, no pós-operatório a nossa paciente ficou paraplégica.

\section{Referências}

1 Garg N, Sampath S, Yasha TC, Chandramouli BA, Devi BI, Kovoor JM. Is total excision of spinal neurenteric cysts possible? $\mathrm{Br}$ J Neurosurg 2008;22(2):241-251

2 Cai C, Shen C, Yang W, Zhang Q, Hu X. Intraspinal neurenteric cysts in children. Can J Neurol Sci 2008;35(5):609-615

3 Kimura H, Nagatomi A, Ochi M, Kurisu K. Intracranial neurenteric cyst with recurrence and extensive craniospinal dissemination. Acta Neurochir (Wien) 2006;148(3):347-352, discussion 352

4 Shenoy SN, Raja A. Spinal neurenteric cyst. Report of 4 cases and review of the literature. Pediatr Neurosurg 2004;40(6):284-292

5 Zenmyo M, Ishido Y, Yamamoto T, et al. Intradural neurenteric cyst-two case reports of surgical treatment. Int J Neurosci 2010; 120(9):625-629

6 Agrawal D, Suri A, Mahapatra AK, Sharma MC. Intramedullary neurenteric cyst presenting as infantile paraplegia: a case and review. Pediatr Neurosurg 2002;37(2):93-96

7 Abe K, Oyama K, Mori K, Ishimaru S, Eguchi M, Maeda M. Neurenteric cyst of the craniocervical junction-case report. Neurol Med Chir (Tokyo) 1999;39(12):875-880

8 Lee SH, Dante SJ, Simeone FA, Curtis MT. Thoracic neurenteric cyst in an adult: case report. Neurosurgery 1999;45(5):1239-1242, $1242-1243$ 
9 Yunoki M, Hirashita K, Gohda Y, Yoshino K, Fujimoto S, Mizobuchi $\mathrm{K}$. True intraspinal neurenteric cyst in the lumbosacral regioncase report. Neurol Med Chir (Tokyo) 2007;47(5):237-239

10 Nalm-ur-Rahman, Jamjoom A, al-Rajeh SM, al-Sohaibani MO. Spinal intradural extramedullary enterogenous cysts. Report of two cases and review of literature. J Neuroradiol 1994;21(4): 262-266

11 Singhal BS, Parekh HN, Ursekar M, Deopujari CE, Manghani DK. Intramedullary neurenteric cyst in mid thoracic spine in an adult: a case report. Neurol India 2001;49(3):302-304 